\title{
The interfering effect of word perception on letter identification
}

\author{
JANICE A. LAWRY \\ University of California, Los Angeles, Los Angeles, California 90024
}

\begin{abstract}
Subjects identified a single lowercase letter in a visual display by pressing one of two buttons. Two letters were assigned to each response. Groups received one of three context conditions: word, nonword, or single-letter displays. In words and nonwords, the flanking letters adjacent to the target varied as to whether they were response compatible or incompatible with the target. Single letters produced faster response latencies than either multiletter condition, and words yielded slower latencies than did nonwords. Items containing an incompatible-response flanking letter produced longer latencies than items containing a compatible flanking letter. Subgroups of subjects with different characteristic processing patterns were identified with a separate test. These subgroups were differentially affected by the context conditions in the letter-identification task. A greater subgroup difference was found in nonwords than in words.
\end{abstract}

Whether or not word context directly influences the perception of letters is a question that has received considerable attention in the psychological literature for nearly a century, beginning with the work of Cattell (1886). Much of the early work suggested that letters were identified more rapidly and with greater accuracy when they appeared in words than when they appeared in nonwords or in singleletter displays (e.g., Miller, Bruner, \& Postman, 1954; Postman, Bruner, \& Walk, 1951; Reicher, 1969).

This so-called "word superiority" effect was later analyzed as the ability to utilize the redundancy of letter sequences or orthographic regularity of the language (Aderman \& Smith, 1971; Miller et al., 1954; Smith, 1969). Consistent with this notion were the findings that letters could be identified equally well in pronounceable, orthographically regular nonwords and real words (Baron \& Thurston, 1973) and that letters in highly redundant nonwords were more accurately identified than letters in low redundant words (Smith, 1969).

In addition to orthographic regularity, pronounceability of the context stimulus also influences performance (Gibson, Osser, \& Pick, 1963; Gibson, Pick, Osser, \& Hammond, 1962), as does positional frequency of the letters in the display (Mason, 1975).

This research was supported, in part, by a grant to David LaBerge from the National Science Foundation (BNS-7904677) and, in part, by grants to the Center for Research in Human Learning, University of Minnesota, from the National Institute of Child Heaith and Human Development (HD-01136) and from the National Science Foundation (BNS-77-22075). Reprint requests should be sent to Janice A. Lawry, Department of Psychology, University of California, Los Angeles, 405 Hilgard Drive, Los Angeles, California 90024.
Estes (1975a, 1975b) has concluded that such factors as these affect response decisions rather than perceptual processes per se. When insufficient stimulus information about the target stimulus exists, subjects may utilize information from the surrounding letters to reduce the number of probable response alternatives. For most words and other orthographically regular displays, this would increase the likelihood of a correct response, but it would have just the opposite effect for displays that violated normal orthographic rules.

Note that the inferential process outlined by Estes (1975a, 1975b) focuses on information from other letters in the display. One might also suggest that the word itself provides a source of information that would allow subjects to infer what letters were presented. This possibility assumes that word perception may precede complete recognition of its constituent letters, a notion that will be explored later.

Suppose that, in the Reicher's forced-choice postcue procedure, a subject is briefly shown the word CASH and is then asked to report whether the letter in the last position was an $\mathrm{H}$ or a $\mathrm{T}$. If CASH was perceived, then the subject can infer that the last letter was an $\mathrm{H}$, knowing that cash contains an $\mathrm{H}$ in that position. Here the inference is based on what the subject knows about the perceived word rather than the perceived letters.

The data of Johnston and McClelland (1974) are consistent with the idea that inferences based on word perception may partially account for the word superiority effect. In this study, one group of subjects were presented with word displays and then asked to make a forced-choice decision about one of the letters in the display. On half of the blocks, the subjects were instructed to try to see the whole word. 
On the other half, they were cued as to which position would contain the target letter and were told to fixate on that position and try to see the letter that would appear there. Performance was superior under the whole-word instructions, suggesting that the inferential process encouraged here allows for more accurate identification than does direct letter perception.

It seems reasonable to expect, however, that responses based on such inferences should take longer to execute than those based on straight readout from activated perceptual letter codes. Unfortunately, there is little data to bear this out, since most of the relevant studies have reported only accuracy measures. One exception is a study by Wheeler (1970), in which letters were identified more accurately when presented in word contexts than when presented alone but response times were faster for the singleletter displays.

In another study that included response times, Estes (1977) found that longer latencies were correlated with a word context advantage over nonwords. At the shortest latencies $(<500 \mathrm{msec})$, nonwords showed a $14 \%$ advantage over words. For latencies between 500 and $1,000 \mathrm{msec}$, an $8 \%$ word advantage appeared, and this increased to $13 \%$ when latencies between 1,000 and $2,000 \mathrm{msec}$ were considered. However, even if these longer latencies do reflect inferential processes, there is no way of determining whether the inferences are based on letter information, word information, or both. At the very least, the wide range of latencies, along with the corresponding variation of word context effects, suggests that subjects probably respond to different sources of information across trials. It would seem that the brief-exposure procedure that includes mixed stimuli forces subjects to be sensitive to a variety of information sources, since no one source is likely to be consistently reliable across trials.

Because different strategies may be differentially useful, depending upon the context, subjects in mixed block designs may have some difficulty in choosing a consistent strategy for the type of information to consider. Data from studies in which probe trials of a different context are inserted into a pure block have consistently shown that subjects do vary their strategies depending upon what they expect to see (Aderman \& Smith, 1971; Carr, Davidson, \& Hawkins, 1978).

Unfortunately, the central question has sometimes been lost within methodological problems and the interpretational difficulties they impose. It would seem, however, that such problems require a refinement of the question. Instead of asking how a word context interacts with letter perception, suppose one asks how word perception interacts with letter perception. This distinction needs to be made, since presenting a word does not guarantee its perception; perceptual events are influenced by many factors besides the stimulus itself. This is clearly supported by the difference obtained between mixed and pure block designs (e.g., Carr, Davidson, \& Hawkins, 1978 ) and the reversal of word/nonword differences brought about by a simple change in instructions (Johnston \& McClelland, 1974).

To the extent that subjects do perceive words, these words may dominate perceptual processing, thereby obscuring, or "masking," the individual letters. One way to characterize the situation is to consider a letter in a word as an embedded figure. Using this embedded-figure analogy, it seems appropriate to also borrow the Gestalt view that the "better" the figure, the more difficult it will be to perceive an individual element making up that figure. The "goodness" of a word figure must be evaluated by reference to its linguistic properties. Factors such as orthographic regularity, pronounceability, and relative familiarity should all contribute to the goodness of the figure. In general, the more wordlike a letter display appears, the harder it ought to be to perceive one of its constituent letters as an independent element.

This interference effect seems at odds with current conceptualizations about word context effects on letter perception (e.g., Henderson, 1975). However, there are at least two findings consistent with the idea. First, Johnston and McClelland (1974) found that under their instructions to attend only to the cued position for purposes of identifying a single letter, subjects given consonant strings did somewhat better than those who received word displays. The second hint of a word interference effect is found in the reaction time data of Estes (1977), previously discussed. Recall that when only the fastest reaction times were considered (those not likely to involve inferential processing), there was a $14 \%$ greater accuracy rate for nonwords than for words!

Given the suggestive pattern of these findings, it was decided to further explore potential word interference effects on letter perception. The experiment reported here had an additional purpose as well, that of evaluating the role of individual differences in processing styles as they might interact with context effects. For greater ease of communication, it was decided to describe the procedures and to present the data bearing on each issue separately. In Part 1, the main context effects on letter identification will be examined. In Part 2, the rationale and outcome of the individual differences analysis will be examined.

\section{PART 1 \\ LETTER IDENTIFICATION IN WORD, NONWORD, AND SINGLE-LETTER DISPLAYS}

In choosing a task that would allow word interference effects to be observed, the objections raised 
against previous procedures were considered. To avoid the development of guessing strategies that depend upon and encourage inferential judgments, it seemed preferable to use a procedure in which guessing was unnecessary by providing adequate stimulus information to the subject. This was accomplished in the present experiment by using relatively long stimulus exposures and switching from accuracy to reaction time measures.

By changing the focus of the question from word context effects to word perception effects, it is desirable to maximize the probability of such perception. With this intent, it was decided to have the context condition as a between-subjects factor, with each group receiving word, nonword, or single-letter displays. It was hoped that providing a consistent context for the word group would encourage the normal functioning of word perception processes. As a further precaution against single-letter processing in the multiletter groups, displays were presented to the right or left of the fixation point so that neither the target letter position nor the context display location was precued. Others have found that such target position uncertainty provided a greater opportunity for context differences to emerge (Carr, Lehmkuhle, Kottas, Astor-Stetson, \& Arnold, 1976; Spector \& Purcell, 1977).

A letter identification trial was as follows: First, a brief warning signal (WS) was displayed in the center of the screen; then the WS was removed and a test display appeared to the right or left of the WS fixation point. An arrow pointed to one of the letters (or the only letter in the case of the single-letter context) in the display. The subject's task was to identify this target letter. The target letter was always one of six letters. Four of these (e.g., response-set letters) had predesignated response assignments: Two were assigned to one response and the other two to a second response. The remaining two letters had no response assignments and served as targets on catch trials.

To gather additional information on the perception of letters in these displays, a within-subjects variable regarding the number and response assignment of response-set letters contained in the display were added to the word and nonword groups. In the neutral condition, only the target letter indicated by the arrow belonged to the response set. In the compatible condition, response-set letters also appeared in one or both positions adjacent to the target, and these nontarget response-set letters had the same response assignment as the target letter. In the incompatible condition, response-set letters appearing adjacent to the target had a response assignment that differed from that of the target. See Table 1 for examples.

These conditions are similar to those used by Eriksen and Eriksen $(1974,1979)$, who presented a target letter with three flanking consonants on each side. These flanking letters were assigned to a response that was compatible, incompatible, or neutral with respect to the target letter. They found that reaction times were faster when flanking letters were assigned to the same response as the target, while reaction times were slower when flanking letters were assigned to a response different from that of the target. A similar pattern of effects might be expected in the present study, but the magnitude of effects would be likely to be less, since only one (and occasionally two) of the several flanking letters belonged to the response set on compatible and incompatible trials. This is in contrast to the compatible and incompatible trials of Eriksen and Eriksen (1974, 1979), in which all six flanking letters were members of the response set.

The addition of this flanker manipulation provided a way of evaluating whether letter information was being processed equally in the word and nonword contexts. However, the main focus on the letter-identification task was to determine the direction of word/nonword differences on letteridentification response times when word perception was unimpaired by procedural restrictions. It was expected that response times would be faster for nonwords than for words. Letters presented in isolation were expected to be identified more quickly than either multiletter condition. The superiority of the single-letter condition might involve nonperceptual factors. As discussed by Eriksen and Eriksen $(1974,1979)$, letters in multiletter displays involve both the time it takes to locate the target as well as the time to identify it. In single-letter displays, the time to locate the target would presumably be less than that for multielement displays.

\section{Method}

Subjects

Ninety native English-speaking college undergraduates served as subjects. All of them were enrolled in an introductory psychology course at the University of Minnesota in which they received two extra credit points for their participation.

\section{Design and Rationale}

For purposes of the letter-identification task, the subjects were assigned randomly to one of three context conditions: words, nonwords, and single letters. The letter-identification task was preceded by two pretests that were administered uniformly to all context groups. The first of these was fashioned after a test used by Hock, Gordon, and Marcus (1974) to evaluate individual differences in processing modes. The results of this pretest were used to identify subsamples of subjects in each context group with different global/local processing dominance and to examine whether or not the context conditions explored in the letteridentification task differentially affected these subpopulations. The full description and results of the individual differences analysis are presented in Part 2. The individual difference task was administered first so as to avoid any differential carryover effects of the three context conditions used in the subsequent letter-identification lask.

The second pretest served as a warm-up for the letteridencitication task. It involved responding to the direction indi- 
cated by an arrow presented in a variable-length string of Xs. The results of this task provided a post hoc check on the equivalency of the letter-identification context groups in terms of subjects' general abilities to identify a target stimulus displayed in noise characters.

\section{Task and Stimulus Materials Description}

Arrow-identification pretest. Test displays ranged from four to eight characters in length. In each display, one of the characters was an arrow pointing either to the right or to the left and the remaining characters were Xs. A small vertical line appeared below one of the characters. When this line appeared below an arrow, the subjects were to respond to the direction indicated by the arrow. If the arrow pointed right, the subjects were to press the right button on their response panels. If the arrow pointed left, they were to press the left button on their response panels. On trials in which the vertical marker appeared below an $\mathrm{X}$ rather than below an arrow, the subjects were not to respond in any overt fashion (i.e., catch trials). See Table 1 for examples.

There were four trials at each of four display lengths $(4,5,6$, and $7 / 8$ characters) for each of the two arrow directions, resulting in a total of 32 test trials in which the vertical marker appeared below an arrow. There were eight catch trials, one at each display length for each type of arrow, in which the vertical marker appeared below an $\mathrm{X}$.

Letter-identification test. For all context conditions, the task was to identify a single letter in the display pointed to by a vertical arrow marker. If this "target letter" was an " $i$ " or a "c," the subjects were to press the left button on the response panel. If the target was an "o" or an " $n$," they were to press the right button on the response panel. On those trials in which the arrow pointed to some other letter in the display, the subjects were not to respond (i.e., catch trial). All displays contained lowercase letters.

Word-context subjects were shown a word on each trial. Target letters indicated by the arrow marker never appeared in the initial or terminal letter positions. Trial types contained compatible, incompatible, and neutral flanking letters, as previously described.

Table 1

Examples of Stimuli Used in Arrow and Letter-Identification Tasks

\begin{tabular}{|c|c|c|c|}
\hline \multirow[b]{2}{*}{ Task } & \multicolumn{3}{|c|}{ Context Condition } \\
\hline & Word & $\begin{array}{l}\text { Non- } \\
\text { word }\end{array}$ & $\begin{array}{l}\text { Single } \\
\text { Letter }\end{array}$ \\
\hline & \multicolumn{3}{|c|}{ Arrow } \\
\hline Right-Hand Trial & $\underset{1}{X} \rightarrow \mathbf{X X X}$ & $\underset{1}{X \rightarrow X X X}$ & $\underset{1}{X \rightarrow X X X}$ \\
\hline Left-Hand Trial & $\underset{1}{\mathrm{XX}} \stackrel{\mathrm{XX}}{1}$ & $\underset{1}{X X} \stackrel{x}{\leftarrow} X$ & $\underset{\mid}{X X \leftarrow X X}$ \\
\hline \multirow[t]{2}{*}{ Catch Trial } & $\underset{1}{X X X} \rightarrow X$ & $\underset{1}{X X X} \rightarrow \mathbf{X}$ & $\underset{1}{X X X} \rightarrow \mathbf{X}$ \\
\hline & \multicolumn{3}{|c|}{ Letter Identification* } \\
\hline Compatible Flanker & $\underset{\uparrow}{\operatorname{mice}}$ & $\underset{\uparrow}{\text { eicm }}$ & $\begin{array}{l}\mathrm{i} \\
\uparrow\end{array}$ \\
\hline Neutral Flanker & $\begin{array}{c}\text { basis } \\
\uparrow\end{array}$ & $\begin{array}{c}\text { sbsia } \\
\uparrow\end{array}$ & i \\
\hline Incompatible Flanker & $\underset{\uparrow}{\text { knit }}$ & $\begin{array}{c}\text { tnik } \\
\uparrow\end{array}$ & $\begin{array}{l}\mathrm{i} \\
\uparrow\end{array}$ \\
\hline Catch Trial & $\underset{\uparrow}{\text { quit }}$ & $\underset{\uparrow}{\operatorname{iuqt}}$ & $\begin{array}{l}\mathbf{u} \\
\uparrow\end{array}$ \\
\hline
\end{tabular}

Note-Target indicated by vertical line or arrow marker beneath stimulus. $\quad$ *See description of stimulus materials for explanation of letter-identification trial types.
Examples of these are provided in Table 1. There was a total of 32 instances of each trial type, eight words at each of four word lengths $(4,5,6$, and $7 / 8$ letters). Each target response-set letter appeared twice at each word length. An additional set of words was selected which contained a " $u$ " or an " $r$ " as well as one or more of the response-set letters. These were used on catch trials with the arrow marker pointing to the "u" or the "r." There were 16 such words in all, 4 at each of the word lengths.

The relative frequencies of all the words were evaluated using the Stimulus Frequency Index (SFI) of Carroll, Davis, and Reichman (1971). SFI values were balanced across word-length subset across trial types, with average values ranging from 53.0 to 53.8 and individual values ranging from 37.6 to 69.3 . The set of stimulus words was split into two sets (A and B) with equal instances of each trial type at each length in each set. Half of the word-context subjects received Set A on Block 1 and Set B on Block 2; the remaining subjects received the reversed sequence. Each set contained 56 unique stimulus words, each presented once for a total of 112 letter-identification test trials. An additional set of practice items was generated which included 16 trials, four instances of each of the trial types, one at each word length.

The nonword-context subjects received compatible, neutral, incompatible, and catch trials like those described for the wordcontext group except that all displays were nonwords. The nonword stimuli were generated by scrambling the letters in the word sets, with the following restrictions: The position of the target letter remained constant and the remaining response-set letters were repositioned so that they remained adjacent to the target letter. Examples are provided in Table 1. Although there was an attempt to make the nonwords nonpronounceable, the inclusion of the vowels and fixed position of the target resulted in some pronounceable items.

The single-letter subjects received single-letter displays. These letters corresponded to the target letters of the word- and nonwordcontext groups and were presented in the exact same location of the screen as the other groups. Since the display contained only a single letter, there were essentially only two kinds of trials for this group, those in which the letter belonged to the response set (test trials) and those in which it did not (catch trials). Consistent with the displays of the other groups, a small arrow pointed to the target letter in each display.

\section{Apparatus}

All stimuli for the arrow- and letter-identification tests were presented on a 38-cm-diagonal television screen driven by a Tektronix 4501 scan converter. A Data General NOVA computer controlled all aspects of stimulus presentation, timing, and data collection. Black lowercase characters were displayed on a white background. Each character was designed with a 7 by 15 dot matrix that measured approximately $9 \times 19 \mathrm{~mm}$ on the screen, subtending $25 \times 38 \mathrm{~min}$ of visual angle. The space between adjacent characters was approximately $2.6 \mathrm{~mm}$.

The subjects were tested individually or in pairs seated in partitioned booths within an experimental room. The television screen in each booth was mounted at eye level and positioned $125 \mathrm{~cm}$ from the edge of the table where the subject was seated. On the table in front of each subject was a response panel containing two response buttons, $25 \mathrm{~cm}$ in diameter. The subjects controlled the onset of each block of trials by pressing one of the buttons in response to the prompt "ready" on the screen.

\section{Procedure}

The subjects were informed that the experiment would involve three different tasks. The first task, which tested for individual differences in processing style, is further explained in Part 2. The procedures for conducting the other two tasks are described below.

The second task was the arrow-identification pretest previously described. The trial sequence was as follows: First, a warning 
signal (WS), consisting of three plus signs $(+++)$, was presented in the center of the screen for $750 \mathrm{msec}$; then the WS was removed and the target display was presented slightly below and to the right or left of the WS position after an ISI of $300 \mathrm{msec}$. The subjects were instructed to identify the character above the line marker and to respond only when this character was an arrow, pressing the left button when the arrow pointed left and the right button when the arrow pointed right. The target display remained on until the subject responded or for a maximum duration of $1,500 \mathrm{msec}$. Response latencies exceeding $1,500 \mathrm{msec}$ were regarded as "misses" and not included in the data analysis. The subjects were encouraged to respond as quickly and as accurately as possible. An error message was presented following an incorrect response or following a response whose latency exceeded $1,500 \mathrm{msec}$. The intertrial interval was $1,250 \mathrm{msec}$, during which the screen remained blank. The subjects received one block of 32 test trials.

The final letter-identification task comprised a brief practice set ( 16 trials) followed by two test blocks ( 56 trials each). The subjects were given a brief rest period after the practice and between the test sets. Prior to the start of the practice, the subjects were provided with a stimulus-response assignment card that contained the response-set letters. The card was placed above the buttons on the response panel. The letters " $i$ " and " $c$ " were positioned above the left button and the letters " $o$ " and " $n$ " were positioned above the right button.

This task was described to the subjects as being similar to the previous arrow-identification task. Once again, a WS has to be presented, followed by a stimulus display in which they were told to identify the letter in the display that appeared directly above the arrow marker. It was explained that this target letter would be appearing in a word (word context group), a string of letters (nonword context group), or alone (single-letter context group). If the target was identified as one of the letters on the card, they were to press the corresponding response button. The subjects were also instructed to memorize the response assignments of the letters shown on the card, since the card would be removed following the practice set. The timing and display characteristics of this task were identical to those of the arrow-identification task, with the exception that the subjects were allowed up to $1,750 \mathrm{msec}$ to respond. Latencies exceeding $1,750 \mathrm{msec}$ were regarded as misses.

After the practice, the experimenter removed the stimulusresponse card and administered the two test sets without further interruption. At the end of the second test, the subjects were given a brief explanation of the experiment and thanked for their participation.

\section{Results}

The analyses of the arrow-identification data included group and display length factors. The subjects in the three context groups received identical treatment on this test, and the group factor was included in the analyses as a check on the random subject assignment. Neither the group nor the length factor produced significant effects on latencies or error rates. The mean group latencies and error rates are given in Table 2 . As can be seen there, the three groups produced similar data, thus providing some evidence that the subject assignment had produced roughly equivalent groups.

Next, the letter-identification data were analyzed. Previous work had indicated that word/nonword context differences might be rather small $(<50 \mathrm{msec})$. Because of the large variance in latencies between subjects within groups, this could create somewhat of a problem for the between-subjects design. An ANOVA based on subjects as the unit of observation would be insensitive to small context differences. Alternatively, it was decided to use items as the unit of observation, where "item" refers to a particular target letter in a particular display position. Since this was kept constant across context conditions, items are crossed with the group factor, providing a "within" design that should be more sensitive to small context differences. Item scores were determined by averaging across the 30 subjects within each context group.

First, analyses were conducted on all three groups and included context and block factors with items nested in blocks. The very substantial group effect on response latencies $[\mathrm{F}(2,188)=1245.25, \mathrm{p}<.001]$ was largely due to the total lack of overlap between latencies produced by items in the single-letter context and latencies produced by items in the two multiletter context conditions. The mean latency for each context condition is included in Table 2. All groups showed a decrease in latencies across the two test sets, yielding a reliable Block effect $[F(1,94)$ $=12.23, \mathrm{p}<.001]$.

As evidenced by the values included in Table 2 , there were few errors made in this task. There were no overall significant main effects due to context conditions or blocks, nor did these factors produce a reliable interaction.

After it was shown that target letters are identified over $200 \mathrm{msec}$ faster in single-letter displays than in multiletter ones, the word/nonword differences were more fully analyzed. Flanker type (compatible, neutral, incompatible) and display length $(4,5,6$, $7 / 8$ ) were added to the context and block factors, with items nested within Block by Flanker by Length cells.

Once again, context yielded a reliable main effect $[\mathrm{F}(1,72)=48.32, \mathrm{p}<.001]$. In these multiletter conditions, there was a reliable 33 -msec improvement over test blocks $[\mathrm{F}(1,72)=10.88, \mathrm{p}<.001]$. The type of flanker also had a significant effect on response latencies $[F(2,72)=6.52, p<.01]$. As illustrated in Figure 1, response latencies were shorter in both contexts when one of the nontarget flanking

Table 2

Mean Response Latencies (L) and Percent Errors (E) for Context Groups in Arrow and Letter-Identification Tasks

\begin{tabular}{lllllc}
\multicolumn{1}{c}{ Arrow Task } & & \multicolumn{2}{c}{ Letter Task } \\
\cline { 2 - 5 } Context & $\mathrm{L}$ & $\mathrm{E}$ & & $\mathrm{L}$ & $\mathrm{E}$ \\
\hline Word & 745 & 2.4 & 826 & 2.4 \\
Nonword & 735 & 1.5 & 792 & 2.3 \\
Single Letter & 730 & 2.7 & 573 & 2.4 \\
\hline
\end{tabular}

Notc Mean response latencies are given in milliseconds. 


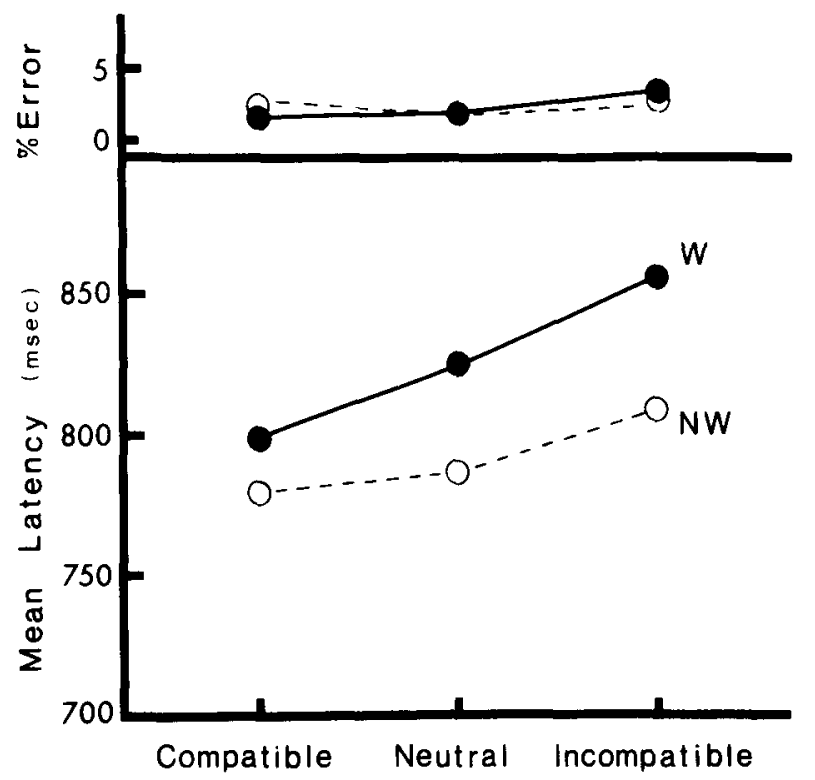

FLANKER TRIAL TYPE

Figure 1. Mean response latency and error rate to identify a single letter in word (W) or nonword (NW) displays as a function of the response assignments of flanking letters. $(N=\mathbf{N}=$ in each context condition.)

letters cued a response that was compatible with the target letter response and response latencies were longer when one of the flanking letters cued a response that was incompatible with the target letter response. Planned comparisons revealed that the difference between compatible and incompatible flanking trials was reliable $[t(72)=3.57, p<.001]$. However, only the incompatible condition was reliably different from the neutral condition $[t(72)=2.19$, $\mathrm{p}<.05]$.

Figure 2 illustrates that response latencies increased with display length in both context conditions. This effect was reliable $[F(3,72)=6.96$, $\mathrm{p}<.01]$. None of the factors analyzed yielded reliable interactions. Furthermore, no single factor or any interaction of factors produced reliable effects on error rates.

\section{Discussion}

The context conditions explored here do affect letter-identification response times. Clearly, letters presented in isolation are identified more quickly than those presented in strings of letters. This finding is consistent with that of Wheeler (1970), in which single letters were identified more rapidly than letters in words when using brief exposure conditions. As previously discussed, this difference may reflect greater ease of target location in the single-letter display (Eriksen \& Eriksen, 1974, 1979). The substantial effect of display length indicates that the greater the number of flanking letters, the slower the response time. This pattern is also consistent with the idea that the greater the number of noise elements in a display, the longer it takes to locate the target.

Of more central concern here, however, are the findings for the multiletter strings. As predicted, words yielded significantly slower response times than did nonwords. Furthermore, the identity of the flanking letters was also an influential determinant. Both word and nonword groups were faster when a compatible response-set letter flanked the target than when an incompatible response-set letter flanked it, thus replicating the general pattern obtained for consonant strings obtained by Eriksen and Eriksen (1974, 1979). Although small, the flanking letter effect was quite reliable, and there were no statistical differences in the magnitude of the effect between word- and nonword-context conditions.

If letter information is equally available in both word and nonword contexts, as is indicated by the flanker data, then why do words produce longer latencies? It would seem that words do not directly interfere with letter information pickup, but rather that they interfere with our ability to utilize this information efficiently. Word information, by virtue of its perceptual dominance, seemingly makes it more difficult to respond to the less salient letter information that is being processed concurrently.

A similar kind of analysis can be applied to a recent study by O'Hara (1980), in which subjects were asked to match pairs of letters. Noise letters which comprised words or nonword anagrams were presented between the target letters. When the noise letters made a nonword, they produced a large in-

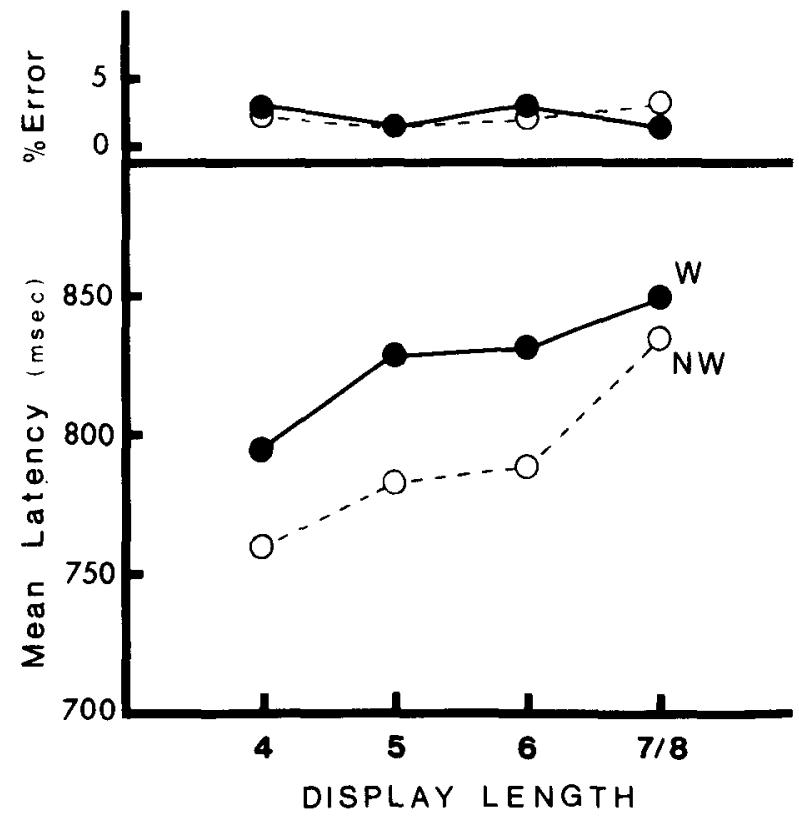

Figure 2. Mean response latency and error rate to identify a single letter in word (W) or nonword (NW) displays as a function of the number of characters in the display. 
crease in response times. In contrast, when the noise letters comprised a familiar word, there was little or no interference relative to the no-noise condition. In this particular design, the perceptual organization of the word stimulus seemingly "masks" its component letters, thus protecting them from interfering with the target letters.

Such effects as that reported here as well as those reported by O'Hara (1980) further support the idea that familiar words are perceptually dominant over letters. There is some evidence to suggest that such dominance is mediated by early-arriving global information. In a picture-naming task, Rayner and Posnansky (1978) found that subjects responded faster when the display contained a label correctly identifying the object or a nonword that preserved the overall shape and first and last letters of the correct label ("hcnre" for "horse"). The facilitation observed for the globally similar nonwords occurred only when the labels were presented for very short durations. At longer durations, slower arriving detail information concerning the inappropriate letters presumably cancels the initial facilitatory effect of the previous global whole-word information.

Consistent with this data, LaBerge and Lawry (in press) have proposed that word codes are initially activated by global information from the display. Attention is then drawn to the code of the highest activation, and further incoming detail or "local" information is matched for consistency with this code. This global/local model of word processing has certain features in common with the general model of visual perception proposed by Lockhead (1972). According to this model, all visual stimuli are thought to be processed first as a "blob" on a holistic basis. Subsequent component processing is said to occur only when the task requires it.

It seems functionally advantageous to construct a processing system that is most sensitive to earlyarriving higher level information and that focuses on lower level information only when a problem arises or the task demands it. For example, in reading, we are less aware of the words than we are of their meanings, and we are even less aware of the letters comprising the words until we encounter an unfamiliar or misspelled word which may require that we attend to the letter information in more detail.

\section{PART 2 \\ ANALYSIS OF INDIVIDUAL DIFFERENCES ON CONTEXT EFFECTS}

In both linguistic (e.g., Rayner \& Posnansky, 1978) and nonlinguistic stimuli (Keuss, 1977; Lockhead, 1972; Navon, 1977), global attributes appear to have perceptual primacy and dominance over detailed local features. However, there may be individual differences which modulate this pattern (Hock, 1973; Hock \& Marcus, 1976). At one extreme there are people whose processing style is dominated by holistic analyses, while at the other extreme there are people whose processing style is dominated by feature analyses. Hock (1973) has labeled these people "structural processors" and "analytical processors," respectively.

Structural processors appear to be more sensitive to contextual fields, or the "global" characteristics of a stimulus, whereas analytical processors appear to be more sensitive to critical features, or the "local" characteristics of a stimulus. Consistent with this analysis, Hock, Gordon, and Marcus (1974) found that structural, or "global-dominant," processors were more impaired than were analytic, or "local-dominant," processors in a letter-detection task in which the target was embedded in a complex geometric pattern. This finding is of particular interest here, since a letter has been characterized as an embedded figure in a word context. To the extent that words and nonwords differ in the salience of their global attributes, it seems reasonable to expect that these two contexts may have a differential influence on response latencies in structural and analytical processors. To explore this possibility, subjects were given a task, fashioned after that used by Hock, Gordon, and Marcus (1974), which measured the effects of both rotation and embedding conditions on letter detection. Analytic, or localdominant, processors and structural, or globaldominant, subjects were to be determined from the results of this test so that context effects could be further evaluated for these subgroups.

\section{Method}

\section{Stimulus Materials and Trial Types}

Three kinds of stimuli were used, normally oriented letters, letters rotated $180 \mathrm{dcg}$, and letters embedded in geometric patterns. Fifteen uppercase letters were selected as targets, all of which resulted in different configurations when rotated $180 \mathrm{deg}$. Embedded figures were constructed by taking a target letter and camouflaging it by introducing symmetries, closures, and continuities in the manner described by Hock, Gordon, and Marcus (1974).

On each trial, the subject was shown a target letter and a stimulus display. The subject was asked to determine if the letter shown also appeared in the stimulus display. There were three kinds of trials in which the target letter was identical to or contained in the stimulus display: (1) normal trials, in which the target and stimulus display were identical letters presented in their normal orientation; (2) rotated trials, in which both the target and stimulus display were identical letters rotated $180 \mathrm{deg}$ from their normal orientation; and (3) embedded trials, in which the target letter was presented in its normal orientation and also appeared embedded in the stimulus display. There were three corresponding trial types in which the target letter did not match the letter contained in the stimulus display. Examples of the stimulus materials used in these six types of trials are provided in Figure 3.

Each of the 15 target letters appeared once in each of the six kind of trials, making a total of 90 test trials. An additional set 

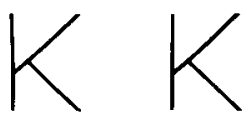

NORMAL
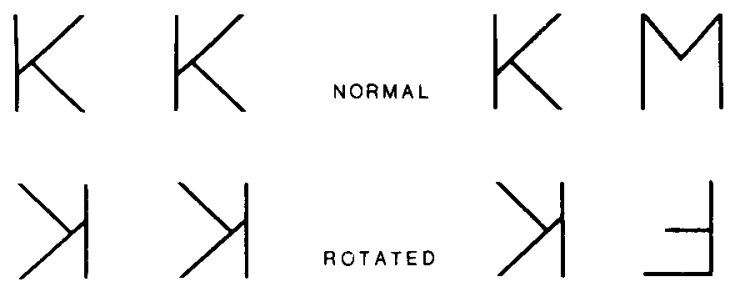

ROTATED
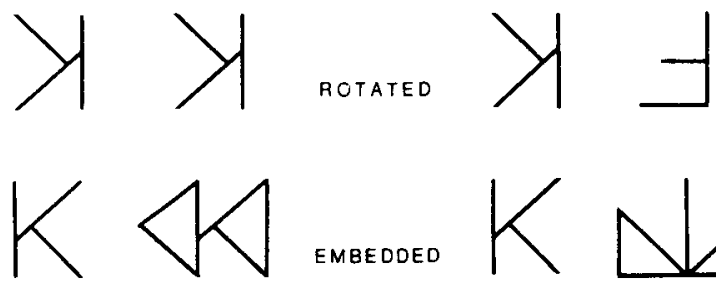

PRESS RIGHT

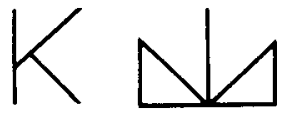

PRESS LEFT

Figure 3. Examples of target and test pattern stimuli used in the individual difference test. See text for full description.

of practice items, exclusive of test materials, was generated. There were two instances of each of the six trial types included in this practice set.

\begin{abstract}
Apparatus
All stimuli were presented on a 42-cm-diagonal Hewlett Packard CRT-P ${ }_{3}$ screen driven by a Data General NOVA computer. The screen was positioned at eye level at a distance of $63 \mathrm{~cm}$ from the edge of the table where the subject was seated. All remaining aspects of stimulus presentation and response collection were controlled by the NOVA computer. White uppercase letters, which measured approximately $22 \times 40 \mathrm{~mm}$ and subtended $4 \times 3.63 \mathrm{deg}$ of visual angle, were used for test materials. Testing conditions were similar to those described in Part 1.
\end{abstract}

\title{
Procedure
}

The test for individual differences consisted of a practice set (12 trials) and a test set $(90$ trials). One $15-\mathrm{sec}$ rest period was given halfway through the test set.

Each trial began with the presentation of the target letter in the upper left quadrant of the screen. After $100 \mathrm{msec}$ had elapsed, the test pattern appeared in the lower right quadrant of the screen. Both the target and test display remained on until a response was made or for a maximum duration of $2,500 \mathrm{msec}$. Response latencies exceeding $3,000 \mathrm{msec}$ were regarded as misses and were not included in the data analysis. An error message was presented following incorrect responses and misses. The intertrial interval was $1,250 \mathrm{msec}$, during which the screen remained blank.

Prior to the practice, the subjects were shown a poster illustrating the six kinds of trial types previously described, and each type was explained by the experimenter. The subjects were instructed to press the right button if the target was identical to the test pattern (i.e., normal and rotation matching trials) or if the target was contained in the test pattern (i.e., embedded trials). They were instructed to press the left button if the target was not the same as or contained in the test pattern. The subjects were given the opportunity to ask questions concerning the task before and after the practice set. If a subject appeared to have misunderstood the instructions and made six or more errors during the practice, the trial types were reexplained and the practice set was rerun. The subjects were encouraged to respond as quickly and as accurately as possible.

\section{Results and Discussion}

\section{Individual Differences Test}

The data from the individual differences test were analyzed separately for those test patterns containing

the target (right-hand responses) and those test patterns not containing the target (left-hand responses). Analyses of response latencies and error rates were based on ANOVAs that included group and test pattern condition as factors, with subjects nested in groups. Again, it should be pointed out that the three context groups received identical treatment in this phase, and the group factor is included here as a further check on subject assignment. However, the latency and error rate analyses revealed no reliable group differences for either right- or left-hand data.

Test pattern condition did have a significant effect on right-hand latencies $[\mathrm{F}(2,174)=533.87, \mathrm{p}<.001]$ and error rates $[\mathrm{F}(2,174)=69.51, \mathrm{p}<.001]$. As can be seen from the mean latencies and error rates in Table 3, the rotation condition was slower than the normal condition $[\mathrm{t}(174)=4.12, \mathrm{p}<.001]$ and the embedded condition was slower than both the normal condition $[\mathrm{t}(174)=30.17, \mathrm{p}<.001]$ and the rotation condition $[\mathrm{t}(174)=26.05, \mathrm{p}<.001]$. The error rates generally followed a similar pattern.

The test pattern condition also had a significant effect on left-hand latencies $[\mathrm{F}(2,174)=335.48$, $\mathrm{p}<.001]$ and error rates $[\mathrm{F}(2,174)=9.43, \mathrm{p}<.001]$. These latencies were generally longer than those obtained for the positive test trials, but the overall pattern of condition effects was similar, as can be seen in Table 3.

A partial correlation between the embedded and rotation conditions controlling for normal condition latencies was calculated for each group of subjects. The results were found to be quite variable; in the word group, $r=.30$ (n.s.), in the nonword group, $r=-.01$ (n.s.), and in the single-letter groups, $r=-.74(p<.001)$. These values did not compare favorably with the results obtained by Hock et al. (1974), who found a much stronger positive relationship between rotation and embedded condition effect (i.e., $r=.74(p<.001)$.

Hock et al. (1974) used the magnitude of the rotation effect to separate analytical and structural processors. These two groups were found to have corresponding differences in the magnitude of their embedding effects. Since the rotation and embedding effects were not similarly related here, a decision had to be made about which to use to.identify subgroups with

Table 3

Mean Response Data for All Subjects on Individual Differences Test Match and Mismatch Trial Types

\begin{tabular}{|c|c|c|c|c|c|c|c|}
\hline \multirow{2}{*}{$\begin{array}{c}\text { Response } \\
\text { Type }\end{array}$} & \multirow[b]{2}{*}{ Trial } & \multicolumn{2}{|c|}{ Normal } & \multicolumn{2}{|c|}{ Rotated } & \multicolumn{2}{|c|}{ Embedded } \\
\hline & & $\mathrm{L}$ & $\mathrm{E}$ & $\mathbf{L}$ & $E$ & L & $\mathrm{E}$ \\
\hline & Dioht Hond & 624 & 2.0 & 683 & 3.3 & 1056 & 12.7 \\
\hline Mismatch & Left $H$ and & 828 & 4.5 & 905 & 4.3 & 1220 & 8.1 \\
\hline
\end{tabular}

Note $-L=$ mean response latency (in milliseconds); $E=$ percent error. 
different processing styles. Of the two, the embedded figure effect (EFE) was considered the more appropriate, since such effects have been shown to be correlated with other measures used to identify different perceptual processing styles among people (for a review, see Witkin, Dyk, Faterson, Goodenough, \& Karp, 1962). Furthermore, detecting a target letter in a geometric pattern was considered somewhat analogous to identifying a target letter in a multiletter display, and hence the EFE may be more sensitive for measuring individual differences in the letteridentification task.

Ten pairs of subjects were chosen from each context group. Subjects within a pair were selected for close similarity in normal condition latencies and a disparity in their EFE (i.e., small vs, large). This procedure tended to exclude subjects with very extreme scores. The subjects were equated for their normal condition latencies so as to avoid selecting subgroups that had generally different reaction times. There was no overlap in EFE scores across subjects in the context subgroups.

Table 4 includes the mean right-hand latencies for the three test pattern conditions and the difference between the normal condition and the embedded condition (EFE) for each subgroup within the three context conditions. For convenience, the two subgroups will be referred to as the small embedded figure effect group and the large embedded figure effect group.

A second set of ANOVAs was used to evaluate the effectiveness of the selection procedures in producing genuinely distinct subgroups. The EFE status was now added to the group and test pattern condition factors, with subjects nested within Group by EFE status cells. In the analysis of right-hand response latencies, there was no overall group effect, although the EFE status factor was significant $[F(1,54)$ $=16.17, \mathrm{p}<.001]$. Test pattern condition, however,

Table 4

Individual Differences Test Data for EFE Status Subgroups Within Context Conditions

\begin{tabular}{|c|c|c|c|c|c|c|c|}
\hline \multirow[b]{2}{*}{ EFE } & \multicolumn{2}{|c|}{ Normal } & \multicolumn{2}{|c|}{ Rotated } & \multicolumn{2}{|c|}{ Embedded } & \multirow[b]{2}{*}{ DS } \\
\hline & $\mathrm{L}$ & $\mathbf{E}$ & $\mathrm{L}$ & $\mathrm{E}$ & $\mathbf{L}$ & $E$ & \\
\hline & \multicolumn{7}{|c|}{ Word Context } \\
\hline $\begin{array}{l}\text { Small } \\
\text { Large }\end{array}$ & $\begin{array}{l}606 \\
622\end{array}$ & $\begin{array}{r}.0 \\
1.3\end{array}$ & $\begin{array}{l}646 \\
694\end{array}$ & $\begin{array}{l}6.0 \\
5.3\end{array}$ & $\begin{array}{r}908 \\
1227\end{array}$ & $\begin{array}{r}12.6 \\
8.6\end{array}$ & $\begin{array}{l}302 \\
606\end{array}$ \\
\hline $\begin{array}{l}\text { Small } \\
\text { Large }\end{array}$ & $\begin{array}{l}638 \\
626\end{array}$ & $\begin{array}{l}3.3 \\
2.6\end{array}$ & $\begin{array}{l}\text { No } \\
701 \\
693\end{array}$ & $\begin{array}{r}\text { Jord } \\
.6 \\
5.3\end{array}$ & $\begin{array}{r}\text { ntext } \\
962 \\
1211\end{array}$ & $\begin{array}{r}16.0 \\
9.3\end{array}$ & $\begin{array}{l}324 \\
584\end{array}$ \\
\hline $\begin{array}{l}\text { Small } \\
\text { Large }\end{array}$ & $\begin{array}{l}622 \\
629\end{array}$ & $\begin{array}{l}2.6 \\
2.6\end{array}$ & $\begin{array}{l}\text { Sing } \\
639 \\
706\end{array}$ & $\begin{array}{l}\text { Lette } \\
1.3 \\
4.0\end{array}$ & $\begin{array}{c}\text { Ontext } \\
916 \\
1185\end{array}$ & $\begin{array}{l}18.3 \\
11.3\end{array}$ & $\begin{array}{l}294 \\
566\end{array}$ \\
\hline
\end{tabular}

Note-See text for explanation of subgroup selection. $L=$ mean response latency (in milliseconds): $E=$ percent error; $D S=$ mean of the EFE difference scores.
Table 5

Arrow and Letter-Identification Data for EFE Status Subgroups Within Context Conditions

\begin{tabular}{|c|c|c|c|c|}
\hline \multirow[b]{2}{*}{$\mathrm{EFE}$} & \multicolumn{2}{|c|}{ Arrow Task } & \multicolumn{2}{|c|}{ Letter Task } \\
\hline & $\mathbf{L}$ & $\mathbf{E}$ & $\mathbf{L}$ & $\mathbf{E}$ \\
\hline & \multicolumn{4}{|c|}{ Word Context } \\
\hline Small & 736 & 3.2 & 817 & 2.3 \\
\hline \multirow[t]{2}{*}{ Large } & 742 & .9 & 845 & 2.5 \\
\hline & \multicolumn{4}{|c|}{ Nonword Context } \\
\hline Small & 686 & 1.6 & 762 & 3.4 \\
\hline \multirow[t]{2}{*}{ Large } & 794 & 1.0 & 819 & 1.6 \\
\hline & \multicolumn{4}{|c|}{ Single-Letter Context } \\
\hline Small & 743 & 1.9 & 566 & 2.2 \\
\hline Large & 756 & 3.4 & 587 & 2.1 \\
\hline
\end{tabular}

Note $-L=$ mean response latency (in milliseconds); $E=$ percent errors.

continued to have the largest effect on response latencies $[\mathrm{F}(2,108)=901.80, \mathrm{p}<.001]$, and, as expected by our selection procedures, EFE status interacted significantly with test pattern condition $[F(2,108)=87.29, p<.001]$. No other interactions were statistically reliable.

Although the left-hand response latencies were not considered in the selection procedures, an analysis of these data revealed a pattern that was identical to that obtained for the right-hand latencies.

\section{Arrow Pretest}

The arrow-identification and letter-identification data were not reanalyzed for these EFE subgroups within the context conditions. The mean latency and error rates for all EFE subgroups are included in Table 5. The analysis of the arrow data showed the EFE factor to have an overall effect on response latencies $[F(1,54)=4.81, p<.05]$. Subjects with small EFE status were an average $43 \mathrm{msec}$ faster than subjects with large EFE status. No other factor produced a reliable effect on the arrow-identification latencies.

\section{Letter-Identification Test}

Including all three context conditions in the letteridentification ANOVA yielded significant main effects due to context $[\mathrm{F}(2,188)=1068.41, \mathrm{p}<.001]$ and EFE status $[F(1,94)=63.73, p<.001]$. These factors were also found to produce a significant interaction $[\mathrm{F}(2,188)=8.43, \mathrm{p}<.001]$. The mean latencies and error rates for EFE subgroups with each of the context conditions are included in Table 5 . It can be seen here that the difference between the EFE subgroups was twice as large in the nonword context as either the word or single-letter contexts.

There was also a small overall improvement over blocks $[F(1,94)=13.24, p<.01]$. Furthermore, the block factor produced a marginally significant interaction with EFE status $[F(1,94)=4.27, p<.05]$, 
The improvement for the large EFE subgroups was somewhat greater than that for the small EFE subgroups. No other interactions were statistically reliable. Error rates were consistently low for all conditions, with none of the factors producing reliable main effects or interactions.

As before, the word and nonword context groups were examined further for flanker and display length effects. Consistent with the earlier analyses, word context produced reliably longer latencies than did nonword context $[\mathrm{F}(1,72)=55.37, \mathrm{p}<.001]$. There were also similar main effects due to blocks $[F(1,72)$ $=15.60, \mathrm{p}<.001]$, display length $[\mathrm{F}(3,72)=7.50$, $\mathrm{p}<.001]$, and flanker type $[\mathrm{F}(2,72)=5.84, \mathrm{p}<.01]$. However, unlike the previous analysis, the flanker condition produced a significant interaction with context $[\mathrm{F}(2,72)=5.19, \mathrm{p}<.01]$. As illustrated in Figure 4, both the facilitatory effect of the compatibleresponse condition and the inhibitory effect of the incompatible-response condition were larger in the word context than in the nonword context.

The EFE status also produced a significant effect $[\mathrm{F}(1,72)=56.65, \mathrm{p}<.001]$, and this factor produced a significant interaction with context $[F(1,72)=7.78$, $\mathrm{p}<.01]$. As illustrated in Figure 5, large EFE subjects were generally slower than small EFE subjects, but this difference was greater in the nonword context than in the word context.

The EFE factor also interacted with blocks $[F(1,72)$ $=3.63, \mathrm{p}<.05$ ], yielding the pattern previously described for all three context conditions. There was

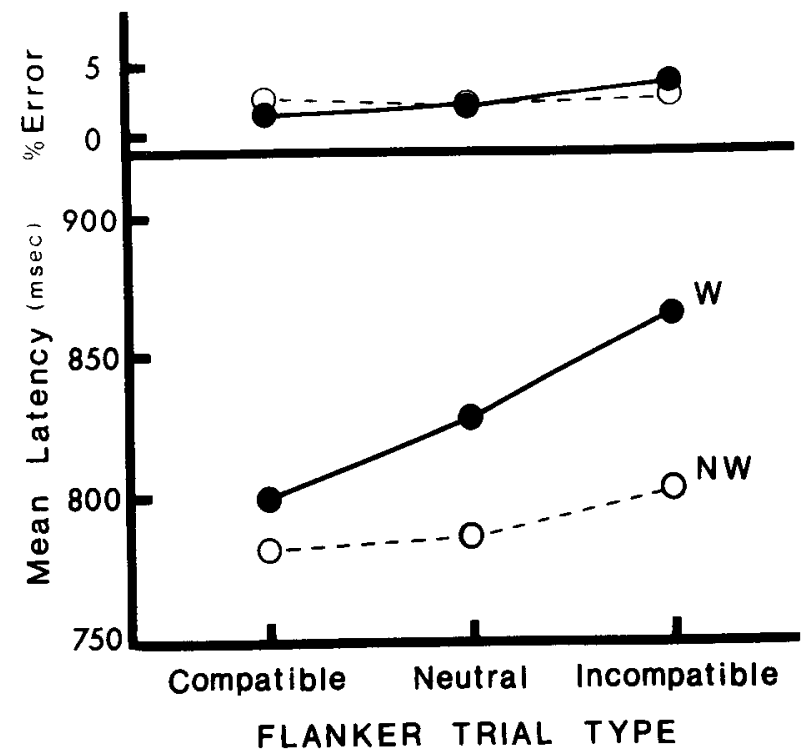

Figure 4. Mean response latency and error rate to identify a single letter in word (W) and nonword (NW) displays for subjects selected for individual differences analysis as a function of the response assignments of flanking letters. $(N=20$ in each context condition.)

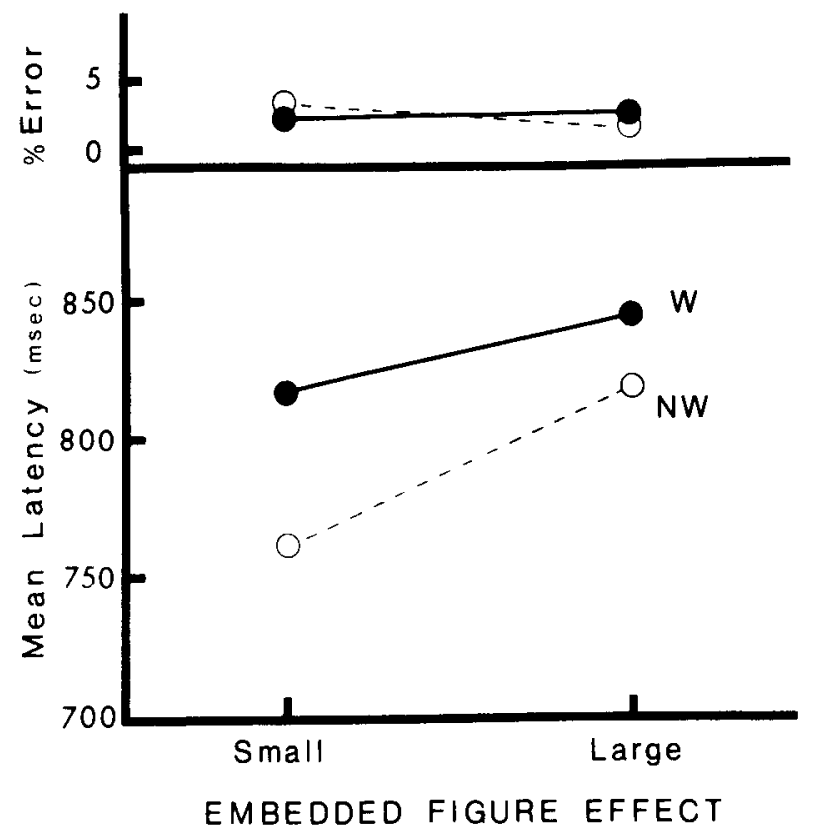

Figure 5. Mean response latency and error rate for subgroups selected on the basis of differential embedded figure effects. See text for explanation of subgroup selection.

also a marginal three-way interaction of context, EFE status, and display length $[F(3,72)=4.06$, $\mathrm{p}<.05]$. This was due to the slight drop in latencies at the third display length for two subgroups while the remaining two showed consistent increases in latency with increasing display length.

To summarize, subjects vary widely in their abilities to detect a target letter embedded in a complex geometric pattern. Subjects who are slower to detect such embedded targets are also slower to identify a single element in a multielement display. This is true when the target is categorically different from the noise or flanking elements, as in the arrow task, and also when the target is categorically similar to the noise characters, as in the letter-identification task.

Large EFE subjects are thought to have a globaldominant processing mode, whereas small EFE subjects are thought to have a local-dominant processing mode. The separation of subjects into globaldominant and local-dominant processors should be considered only a useful abstraction of true individual differences. The distribution of EFE scores obtained here is not bimodal, nor does it appear so in the data reported by Hock, Gordon, and Marcus (1974). The majority of EFE difference scores fell within a square-wave distribution, with a few extreme, large EFE scores trailing off at the upper end. Such a pattern suggests that there is a continuous variation in global/local weightings across individuals.

The interaction between processing style and context condition indicates that the relative amounts of 
global and local processing are not only a function of individual differences, but also the characteristics of the display. This has been illustrated also by Hoffman (1980) for large letters comprised of small ones. When either the small or the large letters were physically distorted, the information provided by the nondistorted level was harder to ignore.

In the present study, stimuli that are "globally rich" or have "globally meaningful" attributes, such as words, may encourage greater global processing in all subjects. Stimuli in which the global attributes are less familiar or meaningful, such as nonwords, allow individual differences to play a greater role in performance.

\section{GENERAL DISCUSSION}

The effect of word perception on letter-identification response times was investigated using relatively long stimulus durations. Letters were identified more slowly in words than in either nonwords or singleletter displays. This word inferiority effect needs to be reconciled with the widely reported word superiority effect that Reicher (1969) and others found when using brief exposures and accuracy measures.

One account for these seemingly incongruent patterns of results stems from the idea that words provide an additional source of information which allows word perception to precede letter perception (LaBerge \& Lawry, in press). This information is thought to be global in nature and dominates processing because of its early arrival in contrast with the later-arriving local or component feature information necessary to identify letters (Rayner \& Posnansky, 1978). During a brief exposure to a word stimulus, sufficient global processing may result in word recognition, even though all the constituent letters may not have been clearly perceived. Such word perception then provides a basis for accurate inferences to be made about the letters (e.g., Johnston \& McClelland, 1974). Nonwords have less familiar global attributes, and these cannot activate a familiar whole-item code. Hence, correct letter identification in nonwords as well as in single-letter displays results from direct readout from activated perceptual letter codes. Letter perception presumably depends more upon the local feature analysis which may not be completed under short exposure conditions, thereby reducing the accuracy of nonword and single-letter conditions relative to words.

Although accuracy of letter identification is apparently superior for words under short exposure conditions, reaction times are not correspondingly faster (e.g., Wheeler, 1970). However, this pattern is consistent with the proposed explanation, since inferences based upon word perception are likely to take longer than responses based upon direct readout from the perceptual system, thus accounting for the correspondence between increasing response latencies and increasing word advantage effect noted by Estes (1977). In the present study, however, the use of long stimulus exposure times makes it unlikely that responses were based upon inferences. Why then does the word/nonword response time difference persist? As in the brief exposure condition, the early-arriving global information is expected to initiate word-code activation. This early activation of a meaningful word code may attract attention away from the letters even though the task requires attention to focus on the target letter. This competition for attention between the word code and the target letter code may account for the increased response times in word displays over nonword displays.

Consequently, while word perception does not interfere with letter information pickup, it does indirectly interfere with letter perception by rendering letter information less salient in the system. This is hardly a new idea; in 1897, Pillsbury described it in this way: "The rush of [word] recognition may be so violent that the letters themselves are entirely neglected, ... forgotten, or not seen at all. We are interested only in the word which is the unit of experience. ... When we get there we are so little concerned with the particular sensations that gave rise to it that we pay no attention to them'" (p. 378).

Interestingly, a similar pattern of results has been reported by Klein (1978) for a related phenomenon, the "object superiority effect." Under brief-exposure conditions, line segments are identified with greater accuracy when displayed as an integral part of an object context as opposed to being presented alone or in a field of similar elements that do not comprise a meaningful object. When longer stimulus durations were used and response times measured, Klein found that accuracy and latency measures did not coincide. Consistent with the word/nonword difference reported here, object context displays were found to produce longer latencies than did the nonmeaningful context. Although the effect was small $(27 \mathrm{msec})$, it was reliable.

These data seem to add further support to the idea that global or configural attributes of a display effectively mask their component elements by dominating perceptual processing. It is important to note, however, that this masking effect is not due to direct interference of component information processing. The flanker data clearly show that nontarget letter information is processed as well in words as in nonwords.

Finally, there are individual differences operating here. Some subjects are more impaired by irrelevant contextual information than are others. In particular, global-dominant processors are consistently slower to respond to a single element in a multielement dis- 
play than are local-dominant processors. Such individual differences were most noticeable here in the nonword condition. In words, where the global attributes are more familiar and serve to activate a meaningful perceptual word code, a global-dominant processing pattern emerges for all subjects, although some slight individual differences remain.

The novel pattern of word/nonword response latencies obtained here, together with the prominent role of individual differences, would seem to encourage a close reexamination and reinterpretation of previous findings of contextual effects in the traditional briefexposure studies of Reicher (1969) and others.

\section{REFERENCES}

Aderman, D., \& Smith, E. E. Expectancy as a determinant of function units in perceptual recognition. Cognitive Psychology, 1971, 2, 117-129.

Baron, J., \& Thurston, I. An analysis of the word superiority effect. Cognitive Psychology, 1973, 4, 207-228.

Carr, T. H., Davinson, B. J., \& Hawkins, H. L. Perceptual flexibility in word recognition: Strategies affect orthographic computation but not lexical access. Journal of Experimental Psychology: Human Perception and Performance, 1978, 4, 674-690.

Carr, T. H., Lehmkuhle, S. W., Kottas, B., Astor-Stetson, E. C., \& ARNOLD, D. Target position and practice in the identification of letters in varying contexts: A word superiority effect. Perception \& Psychophysics, 1976, 19, 412-416.

Carroll, J. B., Davis, P., \& Richman, B. Word frequency book. New York: American Heritage, 1971.

Eriksen, B. A., \& ERIKsen, C. W. Effects of noise letters upon the identification of target letters in a nonsearch task. Perception \& Psychophysics, 1974, 16, 143-149.

Eriksen, C. W., \& Eriksen, B. A. Target redundancy in visual search: Do repetitions of the target within the display impair processing? Perception \& Psychophysics, 1979, 26, 195-205.

Estes, W. K. Memory, perception, and decision in letter identification. In R. L. Solso (Ed.), Information processing and cognition: The Loyola Symposium. Hillsdale, N.J: Erlbaum, 1975. (a)

Estes, W. K. On the locus of inferential and perceptual processes in letter identification. Journal of Experimental Psychology: General, 1975, 104, 122-145. (b)

Estes, W. K. On the interaction of perception and memory in reading. In D. LaBerge \& S. J. Samuels (Eds.), Basic processes in reading: Perception and comprehension. Hillsdale, N.J: Erlbaum, 1977.

Gibson, E. J., Osser, H., \& Pick, A. D. A study in the development of grapheme-phoneme correspondences. Journal of Verbal Learning and Verbal Behavior, 1963, 2, 142-146.

Gibson, E. J., Pick, A. D., Osser, H., \& Hammond, M. The role of grapheme-phoneme correspondence in the perception of words. American Journal of Psychology, 1962, 75, 554-570.

Henderson, L. Do words conceal their component letters? A critique of Johnson (1975) on the visual perception of words.
Journal of Verbal Learning and Verbal Behavior, 1975, 14, 648-650.

Hock, H. S. The effects of stimulus structure and familiarity on same-different comparison. Perception \& Psychophysics, 1973, 14, 413-420.

Hock, H. S., Gondon, G. P., \& Marcus, N. Individual differences in the detection of embedded figures. Perception \& Psychophysics, 1974, 15, 47-52.

Hock, H. S., \& Marcus, N. The effect of familiarity on the processing of fragmented figures. Perception \& Psychophysics, 1976, 20, 375-379.

Hofrman, J. E. Interaction between global and local levels of a form. Journal of Experimental Psychology: Human Perception and Performance, 1980, 6, 222-234.

Keuss, P. J. G. Processing of geometric dimensions in a binary classification task: Evidence for a dual process model. Perception \& Psychophysics, 1977, 21, 371-376.

KLEIN, R. Visual detection of line segments: Two exceptions to the object superiority effect. Perception \& Psychophysics, 1978, 24, 237-242.

LABERGE, D., \& LAWry, J. A. Explorations of changing perceptual modes due to attentional factors induced by task demands. In D. Sheer (Ed.), Attention: Theory, brain mechanism, and clinical application, in press.

Lockhe ad, G. R. Processing dimensional stimuli: A note. Psychological Review, 1972, 79, 410-419.

Mason, M. Reading ability and letter search time: Effects of orthographic structure defined by single letter positional frequency. Journal of Experimental Psychology: General, 1975, 104, 146-166.

Miller, G. A., Bruner, J. S., \& Postman, L. Familiarity of letter sequences and tachistoscopic identification. Journal of General Psychology, 1954, 50, 129-139.

Navon, D. Forest before trees: The precedence of global features in visual perception. Cognitive Psychology, 1977, 9, 353-383.

O'Hara, W. P. Evidence in support of word unitization. Perception \& Psychophysics, 1980, 27, 390-402.

Pillsbury, W. B. A. A study in apperception. American Journal of Psychology, 1897, 8, 315-393.

Postman, L., Bruner, J. S., \& Walk, R. D. The perception of error. British Journal of Psychology, 1951, 42, 1-10.

Rayner, K., \& Posnansky, C. Stages of processing in word identification. Journal of Experimental Psychology: General, 1978, 107, 64-80.

Reicher, G. M. Perceptual recognition as a function of meaningfulness of stimulus material. Journal of Experimental Psychology, 1969, 81, 275-280.

Spector, A., \& Puncell, D. G. The word superiority effect: A comparison between restricted and unrestricted alternative sets. Perception \& Psychophysics, 1977, 21, 323-328.

Smiтh, F. The use of featural dependencies across letters in the visual identification of words. Journal of Verbal Learning and Verbal Behavior, 1969, 8, 215-218.

Whe eler, D. D. Processes on word recognition. Cognitive Psychology, 1970, 1, 59-85

Witkin, H. A., Dyk, R. B., Faterson, H. F., Goodenovgh, D. R., \& KARP, S. A. Psychological differentiation: Studies of development. New York: Wiley, 1962.

(Received for publication May 21, 1980; accepted revision received September $9,1980$. 